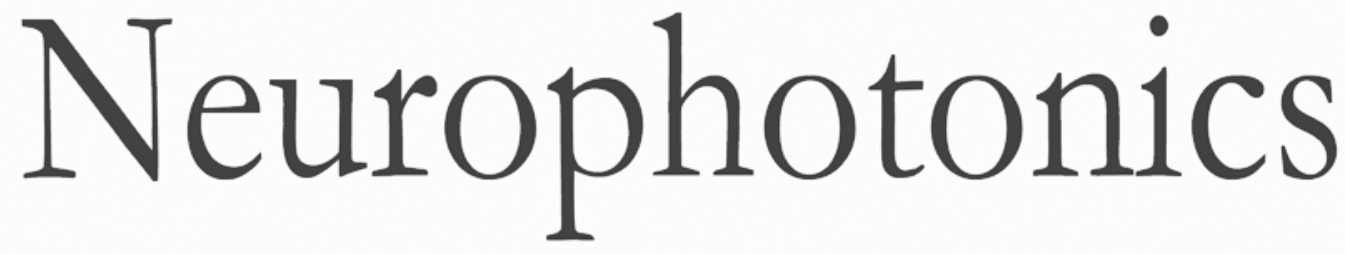

\title{
FNIRS activity in the prefrontal cortex and motivational intensity: impact of working memory load, financial reward, and correlation- based signal improvement
}

Stephen H. Fairclough

Christopher Burns

Ute Kreplin 


\title{
FNIRS activity in the prefrontal cortex and motivational intensity: impact of working memory load, financial reward, and correlation-based signal improvement
}

\author{
Stephen H. Fairclough, ${ }^{a, \star}$ Christopher Burns, ${ }^{b}$ and Ute Kreplin ${ }^{c}$ \\ aLiverpool John Moores University, School of Natural Sciences and Psychology, Liverpool, Merseyside, United Kingdom \\ bUniversity of Warwick, Warwick Manufacturing Group, Experiential Engineering, Coventry, United Kingdom \\ 'Massey University, School of Psychology, Auckland, New Zealand
}

\begin{abstract}
Previous research has demonstrated changes in neurovascular activation of the prefrontal cortex to increased working memory load. The primary purpose of the current paper was to investigate overload of working memory capacity using functional near-infrared spectroscopy (fNIRS) within the framework of motivational intensity theory. A secondary goal was to explore the influence of the correlation-based signal improvement (CBSI) as a method for correcting the influence of systemic variables. In study one, 30 participants (15 female, mean age $=21.09$ years, s.d. $=2.9$ years) performed a verbal version of the $n$-back working memory task under four levels of demand (easy, hard, very hard, and impossible). In contrast to the raw data, CBSI-transformed fNIRS data indicated that neurovascular coupling was highest at hard demand when the task was challenging but success was possible. The second study $(N=30 ; 15$ female, mean age $=22.4$ years, s.d. $=5.3)$ replicated the working memory manipulation with the addition of low versus high levels of financial reward. Analyses of CBSI-transformed levels of oxygenated $(\mathrm{HbO})$ and deoxygenated $(\mathrm{HHb})$ hemoglobin replicated the first study at right lateral regions of the prefrontal cortex (BA46). $\mathrm{HHb}$ _CBSI data were significantly reduced at impossible demand for participants receiving the higher level of financial reward. The study is the first to support predictions from the motivational intensity model using neurovascular data. In addition, the application of CBSI to fNIRS data was found to improve the sensitivity of $\mathrm{HbO}$ and $\mathrm{Hbb}$ to the independent variables. $\odot 2018$ Society of Photo-Optical Instrumentation Engineers (SPIE) [DOI: 10.1117/1.NPh.5.3.035001]
\end{abstract}

Keywords: functional near-infrared spectroscopy; working memory; prefrontal cortex; motivation; reward; signal processing Paper 18009R received Mar. 7, 2018; accepted for publication Jun. 21, 2018; published online Jul. 12, 2018.

\section{Introduction}

Functional near-infrared spectroscopy (fNIRS) is a noninvasive, optical neuroimaging method that provides an index of neurovascular activation but without the need to confine the participant within an apparatus, such as an fMRI scanner. ${ }^{1}$ This technique involves placing a montage of sources and detector optodes upon the scalp, but unlike fMRI, fNIRS is only capable of detecting neurovascular activation in the outermost 10 to $15 \mathrm{~mm}$ of intracranial space. ${ }^{2}$

fNIRS has been applied to the study of higher level cognitive function in the anterior prefrontal cortex. ${ }^{3}$ A number of earlier studies utilized fNIRS in combination with standard experimental paradigms, such as the $n$-back task, to study cognitive function. For example, Ayaz et al., ${ }^{4}$ reported a linear increase of oxygenation in the lateral area of the prefrontal cortex (i.e., BA46) when participants performed a 0-back (i.e., no working memory load) compared with a 3-back (i.e., memorize/update three stimuli) version of the $n$-back task. This pattern of increased oxygenated hemoglobin $(\mathrm{HbO})$ at the prefrontal cortex with higher working memory load has also been observed in a number of later studies. ${ }^{5-8}$ These experiments demonstrated

*Address all correspondence to: Stephen H. Fairclough, E-mail: s.fairclough@ ljmu.ac.uk the potential of fNIRS as an ambulatory technique to tackle applied problems in the field of neuroergonomics, such as the detection of mental overload. ${ }^{9,10}$

The concept of overload in the context of working memory is generally discussed within a finite resource framework, wherein the capacity to store, update, and retrieve information is overwhelmed by the processing demands of the task. ${ }^{11,12}$ Motivational intensity theory ${ }^{13}$ provides a complementary perspective that makes the crucial distinction between the intensity of effort required to meet the demands of the task and the maximum level of effort that a participant is willing to invest in the task. ${ }^{14}$ According to motivational intensity theory, if task demands are fixed and known and participants believe successful performance to be possible, there is a proportionate relationship between mental effort and task demand. However, if demand is increased to the point where success is perceived to be unlikely, effort investment is reduced. This relationship remains unaffected by the importance of task success, which exerts its influence by modulating the upper range of demand levels within which the proportionate relationship holds, i.e., effort investment is sustained at higher levels of demand for those tasks associated with greater success importance. ${ }^{15}$

2329-423X/2018/\$25.00 (C) 2018 SPIE 
Therefore, overload of working memory capacity is associated with three outcomes: poor performance, a perception of low success likelihood, and reduced effort.

The basic predictions of motivational intensity theory have been tested and elaborated through 30 years of psychophysiological research. ${ }^{15}$ However, the availability of neurophysiological data supporting the motivational intensity model is limited. One exception was an EEG study that utilized the $n$-back task to investigate motivational intensity and success importance. ${ }^{16}$ In this study, participants performed three versions of the $n$-back task: 1-back, 4-back, and 7-back, designed to represent easy, hard, and excessive (overload) levels of demand. The authors reported that frontomedial theta activity was significantly higher at hard demand compared with the other two conditions as predicted by motivational intensity theory; the same pattern was found for upper-alpha activity across frontal, central, and parietal sites. When participants were presented with a financial incentive for performance (i.e., increased success importance), upper-alpha activity in the parietal region was significantly suppressed.

One purpose of the current paper is to investigate those hypotheses generated by motivational intensity theory using fNIRS to capture neurovascular activation at the prefrontal cortex in combination with the $n$-back experimental paradigm. However, we know that performance of the $n$-back task causes changes in cardiovascular psychophysiology, which indirectly influence the fNIRS signal and the reliability of inference that can be drawn from fNIRS data. According to the analysis produced by Scholkmann et al., ${ }^{17}$ fNIRS is used to capture cerebral neuronal activity at specific locations evoked by task activity. However, the fNIRS signal is influenced by several systemic confounds, for example, cognitive performance can influence cerebral blood flow and volume at specific sites that are associated with task-related changes in autonomic activity. ${ }^{18}$ The presence of task-evoked and spontaneous confounds on the fNIRS signal is further complicated by potential interactions between cerebral and extracerebral influences. Changes in $\mathrm{CO}_{2}$ levels due to variations in respiratory activity, ${ }^{19}$ fluctuations in mean arterial pressure, and the influence of autonomic mechanisms on cardiovascular variables, such as cardiac output, can affect blood flow at cerebral and extracerebral sites. ${ }^{17}$ When changes in $\mathrm{HbO}$ and deoxygenated hemoglobin $(\mathrm{HHb})$ are observed at cerebral sites, there is potential for occurrence of both false positives (i.e., changes in $\mathrm{HbO}$ and $\mathrm{HHb}$ do not represent task-evoked changes in neurovascular coupling due to neuronal activation) and false negatives (i.e., the influence of systemic activity at both cerebral and extracerebral sites is sufficient to obfuscate the task-evoked changes in neurovascular coupling due to neuronal activation). ${ }^{20}$

At the time of the writing, there are no standard guidelines or specification with respect to signal processing to mitigate the influence of systemic confounds on fNIRS signal. ${ }^{21}$ A number of methods have been proposed, which include: (a) signal processing, e.g., spatial filtering, ${ }^{22}$ Kalman filtering, ${ }^{23}$ and adaptive filtering, ${ }^{24}$ (b) inclusion of additional "short-distance" leads to detect blood flow in the scalp to be subtracted from the fNIRS signal, ${ }^{25}$ and (c) a range of statistical approaches, such as general linear models, ${ }^{26}$ principal component analyses, ${ }^{27}$ wavelet coherence analysis, ${ }^{28}$ autoregressive methods, ${ }^{29}$ and correlative approaches $^{30}$ — see Kamran et al., ${ }^{31}$ for recent review of this topic. The inclusion of short-distance channels represents an effective method for removing systemic confounds; however, many commercial devices do not possess sufficient flexibility to collect these data as a standard feature. ${ }^{21}$ Therefore, one purpose of the current paper is to explore the efficacy of a correlative function ${ }^{32}$ [correlation-based signal improvement (CBSI)] designed to reduce systemic noise and improve signal quality on the sensitivity of fNIRS data in the absence of short-distance channels. $\mathrm{HbO}$ and $\mathrm{Hbb}$ may become positively correlated in the presence of a systemic confound and the CBSI employs a function designed to enhance the negative correlation between both signals, which is characteristic of neurovascular coupling, according to the Balloon model. ${ }^{33}$

This paper describes two experimental studies, both of which use the $n$-back working memory paradigm and incorporate a continuum of task demand from easy to impossible. Both studies will capture fNIRS activation at the prefrontal cortex (BA10, BA46) and operationalize effort as an neurovascular activation. The first study is designed to investigate the motivational intensity hypothesis - that $\mathrm{HbO}$ will be maximized and $\mathrm{HHb}$ will be minimized when working memory demand is high but success is possible. The second study introduces a second independent variable of increased financial incentive in addition to working memory load. It is hypothesized that the presence of a financial incentive will extend the upper limit of working memory demand, where $\mathrm{HbO}$ is maximized and $\mathrm{HHb}$ is minimized.

\section{Study One}

Rationale: The motivational intensity model predicts that participants will invest effort in a proportionate fashion with increased task demand unless the likelihood of success is perceived to be low. This pattern of activation was observed in an earlier study of working memory demand ${ }^{16}$ with respect to EEG measures of fronto-central theta and parietal alpha. This study will replicate the same protocol wherein participants are exposed to a full continuum of working memory demand, from low demand where success is extremely likely to extreme demand when the success likelihood is very low. In correspondence with the motivational intensity model, it is hypothesized that neurovascular activation (i.e., increased $\mathrm{HbO}$, decreased $\mathrm{HHb}$ ) will be highest during the 3-back version of the $n$-back task, when demand is high but success remains possible.

Experimental design: The level of working memory load served as a within-participants manipulation. Four versions of the $n$-back working memory task were used, these were: 1 back (easy and success extremely likely), 3-back (high demand but success possible), 5-back (very high demand and success less likely), and a 7-back (extremely high demand and success extremely unlikely). The levels of working memory load were selected on the basis of an earlier study. ${ }^{16}$

Participants: Thirty participants were recruited from the University population of undergraduate and postgraduate students. The mean age of the participants was 21.09 years (s.d. = 2.9) and the sample included 15 males and 15 females (all white Caucasian). All procedures and measures used in the study were approved by the University Research Ethics Committee and informed consent was obtained from all participants prior to data collection.

Experimental task: A verbal version of the working memory task known as the $n$-back was used in all four conditions. During this task, participants are exposed to a sequential presentation of single capital letters appearing at a rate of $\sim 1$ item every $1500 \mathrm{~ms}$. Stimuli were single capital letters drawn at random from the following group of 12: B, F, G, H, K, M, P, R, S, 
$\mathrm{T}, \mathrm{X}$, and Z. letters were presented centrally in black Arial bold font size 48 against a white background on color monitor at a distance of $60 \mathrm{~cm}$. Participants completed two blocks at each level of working memory demand, each block contained $48 \times 2 \mathrm{~s}$ trials consisting of a 1.5-s stimulus presentation followed by a 0.5 -s interval. At the start of each block, a fixation was present for $4.5 \mathrm{~s}$ prior to onset of the first stimulus and each block lasted for $100 \mathrm{~s}$. The participant must respond to each letter with one of the two possible responses on the keyboard, either the letter is the same as the previous letter (a match) or the letter is different (a nonmatch). The level of demand associated with the $n$-back task is varied systematically by increasing the sequential distance between the current item and the previous item that must be matched. For example, a 1-back version of the task is relatively undemanding as the participant must assess whether the current letter represents a match with the previous letter. However, a 3-back version of the task is more challenging as the current letter must be matched with one that appeared three letters previously. The level of working memory demand rises in a linear fashion with the 5-back and 7-back versions of the task. The E-Prime program was used to create all versions of the working memory task.

Apparatus: The fNIR100 system and cognitive optical brain imaging (COBI) Studio software (Biopac System Inc.) were used for data collection. The source-detector separation was $2.5 \mathrm{~cm}$ and system has a temporal resolution of $500 \mathrm{~ms}$ for one complete data acquisition cycle $(2 \mathrm{~Hz})$. The 16-channel probe was placed on the forehead aligned to Fp1 and Fp2 of the International 10-20 System and rotated so that Fpz corresponded to the midpoint of the probe (see Fig. 1). Areas underlying the 16 channels are right and left superior and inferior frontal gyri; the channels were divided into four regions of interest (ROI), these were located at: left lateral (LL), left medial (LM), right medial (RM), and right lateral (RL) (Fig. 1).

Analyses of fNIRS data: fNIRS data were analyzed offline using fNIRS-Soft software package (BIOPAC Inc.). Variations in light absorption at two different peak wavelengths (730 and $850 \mathrm{~nm}$ ) were used to calculate respective changes of oxygenated $(\mathrm{HbO})$ and deoxygenated $(\mathrm{HHb})$ hemoglobin concentrations (both in $\mu \mathrm{mol} / \mathrm{L}$ ) using the modified Beer-Lambert

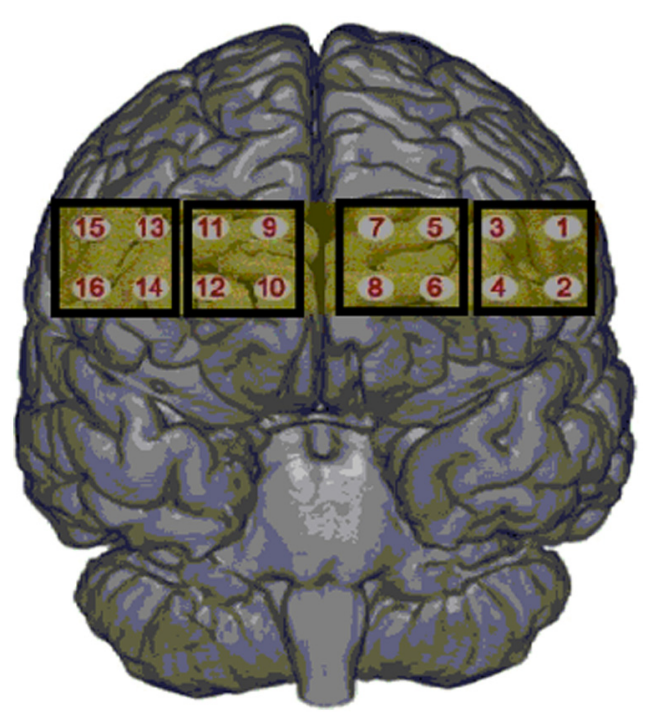

Fig. 1 Placement of fNIRS device and approximate location of 16 channels categorized as four regions of interest.
Law (MBLL). The DPF was set to 6 in line with previous work $^{34}$ and was not adjusted due to the homogenous age range of participants. Raw data were subjected to a slidingwindow motion artifact rejection algorithm, ${ }^{35}$ which is based on the principle that raw optical signals have less variation than segments with motion artifact and saturated channels to remove motion artifacts and saturated channel. A finite impulse response linear phase low-pass filter, with order 20 and cutoff frequency of $0.1 \mathrm{~Hz}$, was applied to attenuate high-frequency noise, respiration, and cardiac effects. ${ }^{35}$ The values of $\mathrm{HbO}$ and $\mathrm{Hbb}$ were calculated relative to the baseline of $30 \mathrm{~s}$ collected during the rest period immediately prior to block onset.

In addition to the mean values from the raw data, mean values for each block were calculated using the CBSI method. ${ }^{32}$ The CBSI formulas were applied to values of $\mathrm{HbO}$ and $\mathrm{Hbb}$ captured at $2 \mathrm{~Hz}$ and subsequently averaged for each $100 \mathrm{~s} \mathrm{block}$ of task activity.

Procedure: Participants were recruited via posters plastered around the University buildings and via email. On arrival, participants were required to read an information sheet about the study and subsequently provide signed consent. After this preliminary phase, participants were seated and provided with a period of practice with all four versions of the working memory task. After training, participants were fitted with the fNIR100 device. Once the apparatus was attached and signal quality was assessed visually via the COBI Studio interface (to identify weak or saturated channels), the experiment began and participants worked through all four versions of the working memory task, which were presented as $2 \times$ blocks of $100 \mathrm{~s}$ duration with a 2 -min rest break between each block. The order of presentation of the four tasks (1-back, 3-back, 5-back, and 7-back) was counterbalanced. The entire duration of the experiment was $\sim 23$ min; upon completion, the apparatus was removed, the participant was thanked, debriefed, and reimbursed for their time $(£ 10 / € 11 / \$ 13)$.

\subsection{Results}

All data were analyzed using SPSS v.23. Participants' performance on the $n$-back task was scored as percentage of correct responses (across both blocks) and subjected to an ANOVA analysis across all four levels of working memory demand. This model revealed a statistically significant effect $[F(3,27)=$ 115.20, $\left.p<0.01, \eta^{2}=0.92\right]$ and posthoc tests confirmed that performance was significantly different between all levels of the $n$-back task $[p<0.01]$; descriptive statistics are shown in Table 1.

Four dependent variables were derived from fNIRS data (HbO, HHb, HbO_CBSI, and HHb_CBSI) and subjected to separate analyses via a 4 (task demand) $\times 4$ (channel) ANOVA within each ROI (LL, left lateral; LM, left medial; RM, right medial; and RL, right lateral). Sphericity was tested via Mauchly's test and the Greenhouse-Geisser adjustment was

Table 1 Descriptive statistics (mean, SD) for performance data in study one expressed as a percentage of correct responses $(N=30)$.

\begin{tabular}{lccc} 
1-back & 3-back & 5-back & 7-back \\
\hline 89.69 & 70.53 & 60.25 & 54.78 \\
{$[4.56]$} & {$[11.44]$} & {$[12.02]$} & {$[9.62]$} \\
\hline
\end{tabular}


Table 2 Results of ANOVA analyses of raw mean $\mathrm{HbO}$ at all four $\mathrm{ROI}$ $(N=30)$.

\begin{tabular}{|c|c|c|c|c|c|c|c|c|}
\hline & \multicolumn{3}{|c|}{ Demand } & \multicolumn{3}{|c|}{ Channel } & \multicolumn{2}{|c|}{$\begin{array}{c}\text { Demand } \times \\
\text { channel }\end{array}$} \\
\hline & $F(3,27)$ & $p$ & $\eta^{2}$ & $F(3,27)$ & $p$ & $\eta^{2}$ & $F(9,21)$ & $p \quad \eta^{2}$ \\
\hline ROI 1 (LL) & 0.75 & 0.53 & - & 1.00 & 0.41 & - & 1.06 & $0.43-$ \\
\hline ROI 2 (LM) & 1.54 & 0.23 & - & 2.74 & 0.06 & - & 1.52 & $0.21-$ \\
\hline ROI 3 (RM) & 3.40 & 0.03 & 0.10 & 1.43 & 0.25 & - & 0.47 & $0.88-$ \\
\hline ROI 4 (RL) & 2.45 & 0.09 & - & 1.28 & 0.29 & - & 0.52 & $0.77-$ \\
\hline
\end{tabular}

made if the Mauchly's test reached significance $(p<0.05)$; posthoc testing was performed via the Bonferroni procedure.

ANOVA analyses of $\mathrm{HbO}$ revealed a significant main effect for task demand at ROI 3 (right medial) only, see Table 2. A similar effect at the same ROI was also found during the analyses of raw HHb (Table 3). Both main effects are shown in Fig. 2. Pairwise comparison tests revealed that mean $\mathrm{HbO}$ was significantly lower during the 1-back version of the $n$-back compared

Table 3 Results of ANOVA analyses of raw mean $\mathrm{HHb}$ at all four ROI $(N=30)$.

\begin{tabular}{|c|c|c|c|c|c|c|c|c|}
\hline & \multicolumn{3}{|c|}{ Demand } & \multicolumn{3}{|c|}{ Channel } & \multicolumn{2}{|c|}{$\begin{array}{c}\text { Demand } \times \\
\text { channel }\end{array}$} \\
\hline & $F(3,27)$ & $p$ & $\eta^{2}$ & $F(3,27)$ & $p$ & $\eta^{2}$ & $F(9,21)$ & $p \quad \eta^{2}$ \\
\hline ROI 1 (LL) & 0.39 & 0.75 & - & 9.99 & $<0.01$ & 0.53 & 1.29 & $0.27-$ \\
\hline ROI 2 (LM) & 0.66 & 0.59 & - & 5.97 & $<0.01$ & 0.18 & 1.36 & $0.26-$ \\
\hline ROI 3 (RM) & 2.89 & 0.05 & 0.09 & 4.37 & 0.01 & 0.21 & 1.57 & $0.19-$ \\
\hline ROI 4 (RL) & 0.16 & 0.92 & - & 1.46 & 0.25 & - & 1.24 & $0.30-$ \\
\hline
\end{tabular}

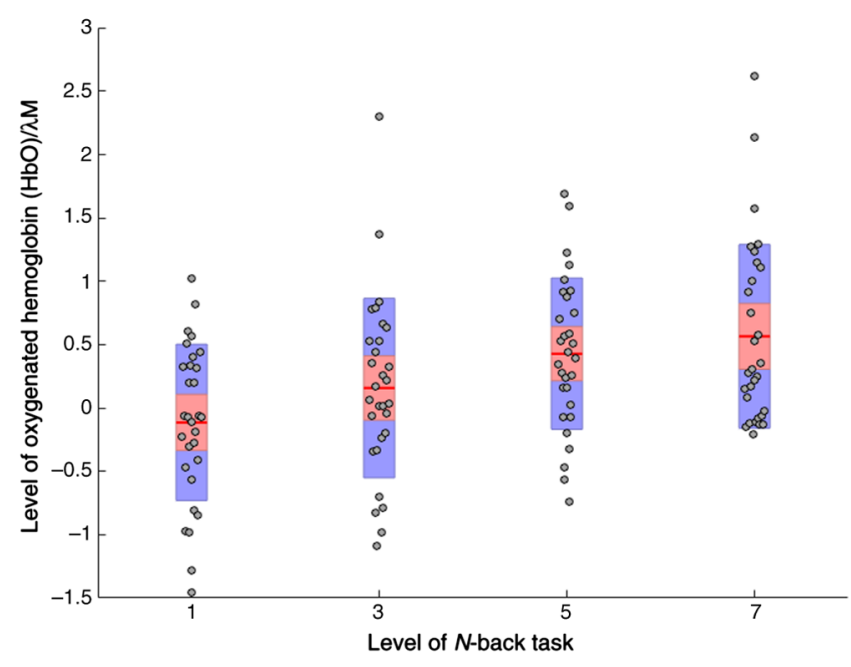

Fig. 2 Levels of $\mathrm{HbO}$ across all four levels of working memory demand (1-back, 3-back, 5-back, and 7-back) at ROI $3(N=30)$. Note: red line $=$ mean, red area $=$ standard deviation, blue bars $=$ $95 \%$ confidence intervals, and gray circles $=$ actual values . with 3-back ( $p<0.05)$, 5-back, or 7-back $(p<0.01)$. Mean levels of $\mathrm{HHb}$ were also significantly lower during the 1-back and 3-back tasks compared with the 5-back $(p<0.05)$.

A number of significant main effects per channel were observed in the analyses of mean HHb. At ROI 1, it was apparent that mean $\mathrm{HHb}$ was significantly lower at channels 2 $(M=-0.25)$ and $4(M=-0.27)$ compared with channels 1 $(M=-0.07)$ and $3(M=-0.03)[p<0.05]$. Mean HHb at channel $8(M=-0.49)$ was significantly lower than channels $5(M=-0.17), 6(M=-0.21)$, and $7(M=-0.12)$ at ROI 2 [ $p<0.01]$. At ROI 3, pairwise comparisons indicated that mean $\mathrm{HHb}$ was significantly lower at channel $11(M=-0.46)$ than channels $9(M=-0.06), 10(M=-0.07)$, or 12 $(M=-0.11)[p<0.01]$.

A second set of data analyses was conducted using the correlative transformation of both mean $\mathrm{HbO}$ and $\mathrm{HHb}^{30}$ as dependent variables (Tables 4 and 5). The analyses of HbO_CBSI revealed a significant effect of working memory demand at ROI 4; pairwise comparisons indicated that $\mathrm{HbO}$ was significantly higher during the 3-back and 5-back versions of the task compared with the 1-back $[p<0.05]$, see Fig. 3. The significant main effect for channel at ROI 1 was identical to the effect described in the previous analyses of raw mean $\mathrm{HbO}$ data. The significant main effect for channel at ROI 2 indicated that $\mathrm{HbO}$ was significantly reduced at channel $7(M=0.21)$ compared with channels $5(M=0.32), 6(M=0.35)$, and $8(M=0.40)$.

The analyses of HHb_CBSI revealed a significant main effect of working memory demand at ROI 4 (Table 5). Bonferroni pairwise comparisons indicated that $\mathrm{HHb}$ was

Table 4 Results of ANOVA analyses of HbO_CBSI at all four ROI $(N=30)$.

\begin{tabular}{|c|c|c|c|c|c|c|c|c|}
\hline & \multicolumn{3}{|c|}{ Demand } & \multicolumn{3}{|c|}{ Channel } & \multicolumn{2}{|c|}{$\begin{array}{c}\text { Demand } \times \\
\text { channel }\end{array}$} \\
\hline & $F(3,27)$ & $p$ & $\eta^{2}$ & $F(3,27)$ & $p$ & $\eta^{2}$ & $F(9,21)$ & $p \quad \eta^{2}$ \\
\hline ROI 1 (LL) & 1.78 & 0.17 & - & 9.31 & $<0.01$ & 0.52 & 1.14 & $0.34-$ \\
\hline ROI 2 (LM) & 1.74 & 0.18 & - & 3.08 & 0.05 & 0.26 & 1.53 & $0.18-$ \\
\hline ROI 3 (RM) & 1.04 & 0.39 & - & 0.99 & 0.42 & - & 1.19 & $0.32-$ \\
\hline ROI 4 (RL) & 2.96 & 0.05 & 0.24 & 1.54 & 0.23 & - & 0.56 & $0.81-$ \\
\hline
\end{tabular}

Table 5 Results of ANOVA analyses of HHb_CBSI at all four ROI $(N=30)$.

\begin{tabular}{|c|c|c|c|c|c|c|c|c|c|}
\hline & \multicolumn{3}{|c|}{ Demand } & \multicolumn{3}{|c|}{ Channel } & \multicolumn{3}{|c|}{$\begin{array}{l}\text { Demand } \times \\
\text { channel }\end{array}$} \\
\hline & $F(3,27)$ & $p$ & $\eta^{2}$ & $F(3,27)$ & $p$ & $\eta^{2}$ & $F(9,21)$ & $p$ & $\eta^{2}$ \\
\hline ROI 1 (LL) & 1.61 & 0.21 & - & 5.68 & $<0.01$ & 0.39 & 2.78 & 0.020 & 0.09 \\
\hline ROI 2 (LM) & 1.12 & 0.36 & - & 1.28 & 0.30 & - & 0.53 & 0.19 & - \\
\hline ROI 3 (RM) & 0.11 & 0.95 & - & 1.83 & 0.17 & - & 1.37 & 0.26 & - \\
\hline ROI 4 (RL) & 3.66 & 0.03 & 0.29 & 0.28 & 0.83 & - & 0.65 & 0.74 & - \\
\hline
\end{tabular}




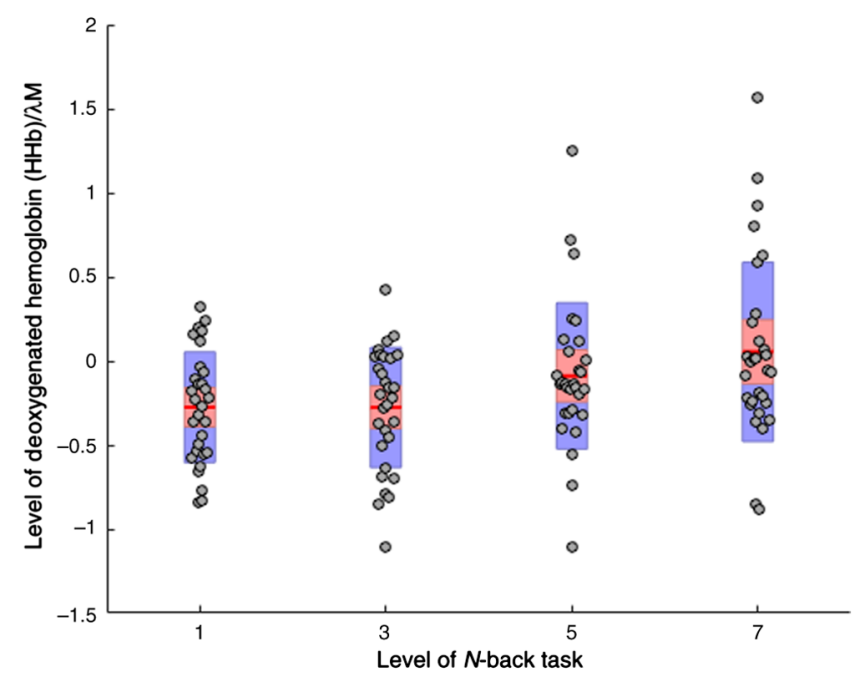

Fig. 3 Levels of $\mathrm{HHb}$ across all four levels of working memory demand (1-back, 3-back, 5-back, and 7-back) at ROI $3(N=30)$. Note: red line $=$ mean, red area $=$ standard deviation, blue bars $=$ $95 \%$ confidence intervals, and gray circles = actual values.

significantly lower during the 3-back compared with either the 1 -back [ $p=0.03$ ] or 5-back [ $p=0.02]$ versions of the task, see Fig. 3. There was a significant main effect of channel at ROI 1, which revealed that $\mathrm{HHb}$ was significantly lower at channels 2 $(M=-0.10)$ and $4(M=-0.11)$ compared with channels 1 $(M=-0.05)$ and $3(M=-0.03)$, respectively. There was also a significant interaction effect at ROI 1; posthoc testing revealed that $\mathrm{HHb}$ was significantly reduced during the 3-back task $(M=-0.20)$ compared with the other three $n$-back conditions: 1-back $(M=-0.07), 5$-back $(M=-0.06)$, and 7-back $(M=$ $-0.08)[t(29)=-2.17, p=0.04]$.

\section{Study Two}

Rationale: Previous research has explored the contribution of motivational intensity and success importance by simultaneously manipulating demand and financial incentive. ${ }^{16,36,37}$ The theory posits that success importance does not directly influence effort investment but increases the range of difficulty levels wherein a proportionate relationship between effort and demand can be observed. ${ }^{15}$ Hence, the presence of an incentive effectively extends the upper limit of task difficulty where effort is invested as a function of task demand. This pattern was observed by Eubanks et al., ${ }^{36}$ who reported increased heart rate at maximum task demand, but only in the presence of a financial incentive. However, Fairclough and Ewing ${ }^{16}$ did not find support for this hypothesis and a general increase of systolic blood pressure in the presence of the financial incentive was observed.

This second study extended study one by introducing a second independent variable of financial reward. Specifically, one group was offered the usual financial reward for participation in the experiment within our institution (£10/\$13.5/€11.3), whereas the second group were told that they would receive an addition level of financial reward (£10) if they met an unspecified performance criterion, allowing them to earn a maximum of $£ 20$ ( $\$ 27 / € 22$ ). The second independent variable was the same four levels of working memory demand (1b, $3 \mathrm{~b}, 5 \mathrm{~b}$, and $7 \mathrm{~b}$ ) used in study one, which were supplemented with a fifth 0 -back $(0 \mathrm{~b})$ condition, i.e., where participants press any button when a letter appears on screen; this condition was included to control for fNIRS changes induced purely by motor responses associated with the task.

It is predicted that $\mathrm{HbO}$ will be maximized and $\mathrm{HHb}$ will be minimized when demand is high and success is likely at the $3 \mathrm{~b}$ version of the task, as observed in study one. It is also hypothesized that a higher level of possible financial reward will increase the range of task difficulty where the proportionate relationship between effort and demand holds, i.e., $\mathrm{HbO}$ will be higher and $\mathrm{HHb}$ will be lower at $5 \mathrm{~b}$ and $7 \mathrm{~b}$ versions of the task for those participants, who can receive a higher level of financial reward.

Experimental design: Five levels of working memory load served as a within-participants manipulation, these were: 0-back, 1-back, 3-back, 5-back, and a 7-back. The level of financial reward for taking part in the study functioned as a betweenparticipants factor, one group were remunerated at $£ 10$, and the other group were told that they could earn $£ 20$ if they met an unspecified performance criterion.

Participants: Thirty participants (15 per reward group) were recruited from the University population of undergraduates and postgraduate students. The mean age of the participants was 22.4 years $($ s.d. $=5.3$ ) and there was an equal number of males and females. With respect to ethnicity, one participant was Asian, but all others were white. Mean age of participants in both reward groups was equivalent (22.6 years versus 22.3 years). All procedures and measures used in the study were approved by the University Research Ethics Committee and informed consent was obtained from all participants prior to data collection.

Experimental task: A verbal version of the working memory task was used in all five conditions. This task is described in the method section for study one.

Apparatus: An fNIRS and COBI data collection suit (Biopac System Inc.) were used for data collection. The set-up for the fNIRS was identical to that used and described in the method section for study one.

Analyses of fNIRS data: The data processing pipeline and analysis protocol were identical to study one. Mean scores for each block of fNIRS data were derived in both raw and transformed forms using the CBSI method. ${ }^{32}$ Full details are provided in the method section for study one.

Procedure: The procedure for the study reproduced the protocol used for study one in all particulars. The only difference was that half of the participants received $£ 20$ for taking part in the study, whereas the other half were provided with $£ 10$ remuneration.

\subsection{Results}

All data were analyzed using SPSS v.23. Participants' performance on the $n$-back task was scored as percentage of correct responses (across both blocks) and subjected to a 2 (reward) $\times 4$ (working memory demand) ANOVA analysis. There was a significant effect for working memory demand $[F(4,25)=125.30$, $\left.p<0.01, \eta_{2}=0.93\right]$. Posthoc tests revealed a significant difference between all four levels of working memory demand. There was no main effect for the between-participants factor of financial reward $[F(1,28)=0.12, p=0.72]$ or any interaction effect. Descriptive statistics for the performance data are shown in Table 6.

The four dependent variables derived from fNIRS ( $\mathrm{HbO}$, $\mathrm{HHb}, \mathrm{HbO} \_\mathrm{CBSI}$, and HHb_CBSI) were subjected to separate 
Table 6 Descriptive statistics (mean, SD) for accuracy data expressed as a percentage of correct responses $(N=30)$.

\begin{tabular}{lcccc} 
& 1-back & 3-back & 5-back & 7-back \\
\hline £10 reward & 89.19 & 70.00 & 59.13 & 49.50 \\
& {$[5.62]$} & {$[9.65]$} & {$[11.22]$} & {$[11.81]$} \\
£20 reward & 90.07 & 71.73 & 59.47 & 50.07 \\
& {$[4.88]$} & {$[10.24]$} & {$[10.75]$} & {$[11.42]$} \\
Combined & 89.61 & 70.84 & 59.29 & 49.77 \\
& {$[5.21]$} & {$[9.82]$} & {$[10.81]$} & {$[11.43]$} \\
\hline
\end{tabular}

Table 7 Main effects of ANOVA analyses of mean $\mathrm{HbO}$ at all four ROI in study $2(N=30)$.

\begin{tabular}{|c|c|c|c|c|c|c|c|c|c|}
\hline & \multicolumn{3}{|c|}{ Reward } & \multicolumn{3}{|c|}{ Demand } & \multicolumn{3}{|c|}{ Channel } \\
\hline & $F(1,28)$ & $p$ & $\eta^{2}$ & $F(4,25)$ & $p$ & $\eta^{2}$ & $F(3,26)$ & $p$ & $\eta^{2}$ \\
\hline ROI 1 (LL) & 0.16 & 0.69 & - & 1.66 & 0.63 & - & 1.66 & 0.20 & - \\
\hline ROI 2 (LM) & 0.05 & 0.83 & - & 0.27 & 0.91 & - & 1.86 & 0.16 & - \\
\hline ROI 3 (RM) & 1.74 & 0.20 & - & 1.16 & 0.35 & - & 3.77 & 0.02 & 0.30 \\
\hline ROI 4 (RL) & 0.78 & 0.38 & - & 0.94 & 0.46 & - & 0.50 & 0.69 & - \\
\hline
\end{tabular}

Table 8 Main effects of ANOVA analyses of mean $\mathrm{HHb}$ at all four $\mathrm{ROI}$ in study $2(N=30)$.

\begin{tabular}{|c|c|c|c|c|c|c|c|c|c|}
\hline & \multicolumn{3}{|c|}{ Reward } & \multicolumn{3}{|c|}{ Demand } & \multicolumn{3}{|c|}{ Channel } \\
\hline & $F(1,28)$ & $p$ & $\eta^{2}$ & $F(4,25)$ & $p$ & $\eta^{2}$ & $F(3,26)$ & $p$ & $\eta^{2}$ \\
\hline ROI 1 (LL) & 0.05 & 0.83 & - & 1.12 & 0.37 & - & 1.03 & 0.40 & - \\
\hline ROI 2 (LM) & 0.00 & 0.99 & - & 0.62 & 0.65 & - & 1.62 & 0.21 & - \\
\hline ROI 3 (RM) & 0.50 & 0.48 & - & 1.04 & 0.41 & - & 1.45 & 0.25 & - \\
\hline ROI 4 (RL) & 0.54 & 0.47 & - & 1.46 & 0.25 & - & 0.61 & 0.62 & - \\
\hline
\end{tabular}

analyses via a 2 (reward) $\times 5$ (task demand) $\times 4$ (channel) ANOVA within each ROI (LL, left lateral; LM, left medial; RM, right medial; and RL, right lateral). Sphericity was tested via Mauchly's Test and the Greenhouse-Geisser adjustment was made if the Mauchly's test reached significance $(p<$ 0.05 ); posthoc testing was performed using the Bonferroni procedure.

The analyses of $\mathrm{HbO}$ revealed only one main effect and no significant interactions. The main effect of channel at RO3 indicated that $\mathrm{HbO}$ was significantly higher at channel 11 $(M=0.01)$ compared with channel $12(M=-0.33)$ (Table 7).

The analyses of $\mathrm{HHb}$ revealed no significant main effects or interactions (Table 8).
Table 9 Main effects of ANOVA analyses of HbO_CBSI at all four $\mathrm{ROI}$ in study $2(N=30)$.

\begin{tabular}{|c|c|c|c|c|c|c|c|c|c|}
\hline & \multicolumn{3}{|c|}{ Reward } & \multicolumn{3}{|c|}{ Demand } & \multicolumn{3}{|c|}{ Channel } \\
\hline & $F(1,28)$ & $p$ & $\eta^{2}$ & $F(4,25)$ & $p$ & $\eta^{2}$ & $F(3,26)$ & $p$ & $\eta^{2}$ \\
\hline ROI 1 (LL) & 1.23 & 0.28 & - & 0.64 & 0.64 & - & 1.94 & 0.15 & - \\
\hline ROI 2 (LM) & 0.47 & 0.50 & - & 0.45 & 0.78 & - & 0.94 & 0.44 & - \\
\hline ROI 3 (RM) & 1.48 & 0.24 & - & 1.07 & 0.39 & - & 0.91 & 0.45 & - \\
\hline ROI 4 (RL) & 0.54 & 0.47 & - & 1.46 & 0.25 & - & 0.61 & 0.62 & - \\
\hline
\end{tabular}

Table 10 Means and standard deviation for $\mathrm{HHb}$ _CBSI for all levels of working memory load at ROI3 between the two reward conditions.

\begin{tabular}{cccccc}
\hline & $0 \mathrm{~b}$ & $1 \mathrm{~b}$ & $3 \mathrm{~b}$ & $5 \mathrm{~b}$ & $7 \mathrm{~b}$ \\
\hline £10 reward & -0.107 & -0.147 & -0.154 & -0.016 & 0.088 \\
& $(0.207)$ & $(0.332)$ & $(0.243)$ & $(0.263)$ & $(0.369)$ \\
\multirow{2}{*}{ £20 reward } & -0.074 & -0.106 & -0.097 & -0.071 & -0.142 \\
& $(0.306)$ & $(0.212)$ & $(0.233)$ & $(0.207)$ & $(0.269)$ \\
\hline
\end{tabular}

Table 11 Results of ANOVA analyses of $\mathrm{HHb}$ CCBSI at all four $\mathrm{ROI}$ in study $2(N=30)$.

\begin{tabular}{|c|c|c|c|c|c|c|c|c|}
\hline & \multicolumn{3}{|c|}{ Reward } & \multicolumn{3}{|c|}{ Demand } & \multicolumn{2}{|c|}{ Channel } \\
\hline & $F(1,28)$ & $p$ & $\eta^{2}$ & $F(4,25)$ & $p$ & $\eta^{2}$ & $F(3,26)$ & $p \quad \eta^{2}$ \\
\hline ROI 1 (LL) & 0.74 & 0.40 & - & 0.12 & 0.97 & - & 2.32 & $0.10-$ \\
\hline ROI 2 (LM) & 0.04 & 0.84 & - & 0.93 & 0.47 & - & 2.26 & $0.11-$ \\
\hline ROI 3 (RM) & 0.27 & 0.61 & - & 1.19 & 0.35 & - & 1.06 & $0.39-$ \\
\hline ROI 4 (RL) & 0.10 & 0.76 & - & 2.72 & 0.03 & 0.33 & 0.95 & $0.43-$ \\
\hline
\end{tabular}

The analyses of HbO_CBSI (Table 9) revealed a significant interaction between demand $\times$ channel at ROI3 $[F(3,26)=$ 2.24, $\left.p=0.01, \eta_{2}=0.68\right]$. Posthoc tests revealed that HbO_CBSI was significantly higher during the 3-back task compared with the 0 -back $[t(29)=-2.43, p=0.05]$ and the 7-back $[t(29)=3.15, p<0.01]$ versions of the task at channel 12 (Fig. 1). Descriptive statistics are shown in Fig. 6.

The analyses of HHb_CBSI revealed an interaction effect between reward and demand at ROI3 $[F(4,25)=2.42, p=$ $\left.0.04, \eta_{2}=0.25\right]$. Posthoc analyses revealed that HHb_CBSI was significantly increased at the 7-back version of the task for those participants in the $£ 10$ group compared with the $£ 20$ group $[t(29)=2.27, p<0.05)$; descriptive statistics are shown in Table 10. There was also a main effect for demand at ROI4 (Table 11), Bonferroni pairwise tests revealed that $\mathrm{HHb}$ CBSI was significantly reduced at the $3 \mathrm{~b}$ and $5 \mathrm{~b}$ versions of the $n$-back task compared with the 0b task $(p<0.05)$ (Fig. 7). 


\section{Discussion}

The initial analyses of uncorrected data in study one revealed a significant linear increase of both $\mathrm{HbO}$ and $\mathrm{HHb}$ from the 1-back to the 7-back version of the working memory task. These findings (Figs. 2 and 3) contradict the predictions of motivational intensity theory, i.e., neural activation would be maximal when demand is high and success remains possible. In addition, minimal levels of $\mathrm{HHb}$ were observed at the 1-back task when demand was lowest, and HHb subsequently increased in a linear fashion, which is counterintuitive from the perspective of neurovascular coupling. The application of the CBSI and subsequent data analyses yielded a different pattern of results (Figs. 4 and 5). The significant effect of working memory demand shifted to right lateral ROI and for both HbO_CBSI and HHb_CBSI, in line with earlier findings for working memory and the $n$-back derived from fMRI data. ${ }^{38}$ In addition, the pattern of significant results was broadly in agreement with the predictions of the motivational intensity model. HbO_CBSI significantly increased and HHb_CBSI showed a significant decrease at the 3-back task when demand was high and success was possible.

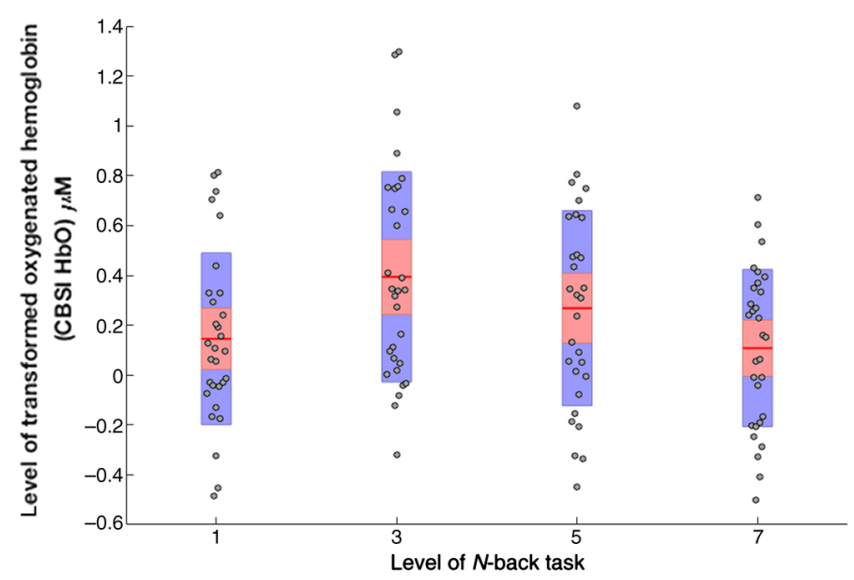

Fig. 4 Levels of HbO_CBSI across all four levels of working memory demand (1-back, 3-back, 5-back, and 7-back) at ROI $4(N=30)$. Note: red line $=$ mean, red area $=$ standard deviation, blue bars $=$ $95 \%$ confidence intervals, and gray circles $=$ actual values .



Fig. 5 Levels of HHb_CBSI across all four levels of working memory demand (1-back, 3-back, 5-back, and 7-back) at ROI $4(N=30)$. Note: red line $=$ mean, red area $=$ standard deviation, blue bars $=$ $95 \%$ confidence intervals, and gray circles = actual values.
The second study was designed to both replicate and extend study one via the inclusion of increased financial reward. As in study one, analyses of transformed data were characterized by a pattern of results that were consistent with the predictions of the motivational intensity model. Both HbO_CBSI at channel 12 (Fig. 6) and HHb_CBSI at ROI4 (Fig. 7) exhibited the expected relationship between neurovascular activation and working memory demand, i.e., maximum $\mathrm{HbO}$ and minimum $\mathrm{HHb}$ during the 3-back version of the task. The interaction between demand and financial reward observed for HHb_CBSI (Table 11) was also consistent with the predictions of motivational intensity theory and earlier work. ${ }^{36}$ All significant experimental effects in the second study were localized to the right side of the prefrontal cortex, approximately at BA10 and BA46.

Three points of convergence emerged from both experimental studies. To the best of our knowledge, our results provided

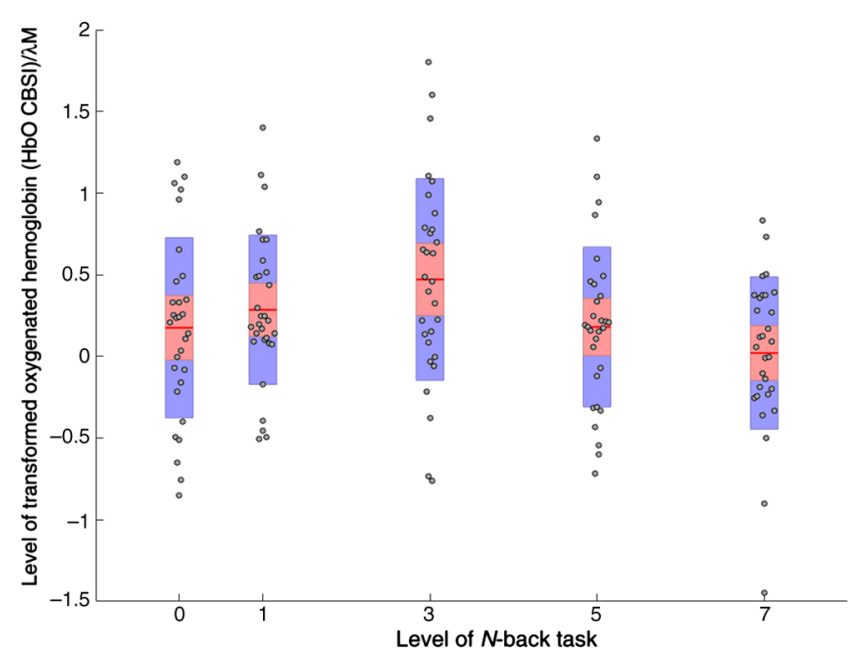

Fig. 6 Mean level of HbO_CBSI at channel 12 across all levels of working memory demand $(N=30)$. Note: red line $=$ mean, red area $=$ standard deviation, blue bars $=95 \%$ confidence intervals, and gray circles $=$ actual values.

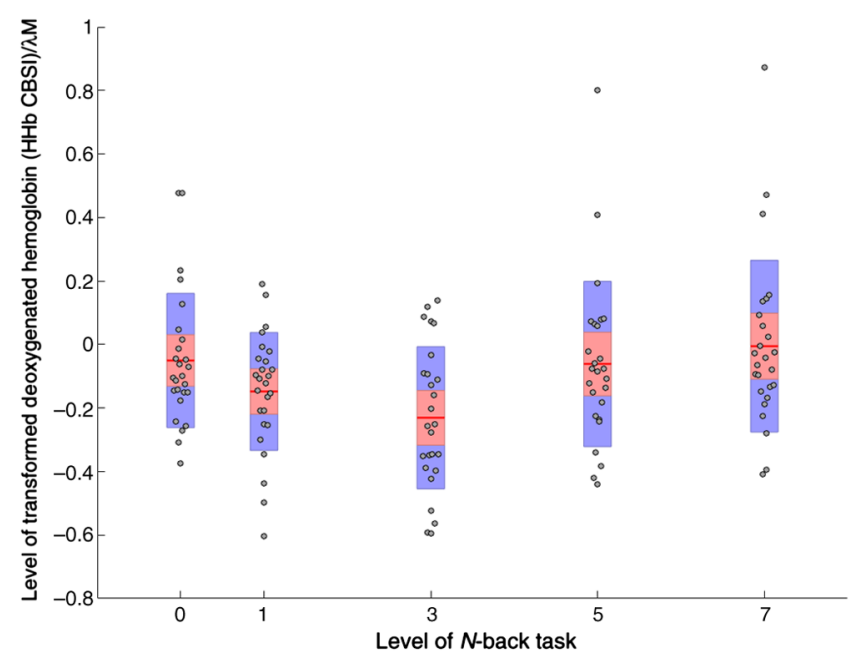

Fig. 7 Mean level of HHb_CBSI at ROI4 across all levels of working memory demand $(N=30)$. Note: red line = mean, red area $=$ standard deviation, blue bars $=95 \%$ confidence intervals, and gray circles $=$ actual values. 
the first formal support for the motivational intensity model using measures of neurovascular measures. Our studies found complementary support for two predictions derived from the model with respect to motivational intensity (both studies) and success importance (study two). Although there is a body of earlier research demonstrating the sensitivity of $\mathrm{HbO}$ to working memory load using the same task, ${ }^{4} \mathrm{HbO}$ increases in a linear fashion from low to high demand on the $n$-back task. The current study extended earlier work by pushing the upper limit of working memory load into the region of "overload" to explore motivational intensity theory. It was also found that all significant effects associated with the manipulation of working memory load were localized at the right medial/lateral channels, which could be approximately localized as BA9 and BA46, respectively. This is significant because this specific area of the prefrontal cortex has been associated with strategic reorganization of working memory content ${ }^{3}$ and previous studies of fMRI revealed activation in this area due to increased demand of the $n$-back task, i.e., see Fig. 1, p. 51 of Owen et al. ${ }^{38}$ The same pattern of activation in response to working memory load has been observed in $n$-back studies using fNIRS ${ }^{5-8}$ Therefore, the specificity of the significant effects associated with $\mathrm{HbO}$ _CBSI and Hbb_CBSI replicates earlier findings from both fMRI and fNIRS experiments, which increases our confidence that the CBSI transformation mitigated the influence of systemic confounds and improved the sensitivity of our data. Finally, the analyses demonstrated that subjecting fNIRS data to the CBSI transformation produced results that contrasted with the original data in two ways: (1) the sensitivity of $\mathrm{HbO}$ and $\mathrm{HHb}$ to independent variables was enhanced with respect to statistical analyses, and (2) the observed pattern of $\mathrm{HbO}$ and $\mathrm{HHb}$ after CBSI transformation was consistent with a priori predictions from the motivational intensity model.

The second goal of the study was to explore the CBSI transformation as a method of reducing the influence of systemic noise when more preferable options such as the presence of short-distance channels are not available. The particular apparatus used in the current study contained only fixed optode holders and we utilized CBSI as a method to mitigate the effects of systemic confounds. The analyses of both studies permitted a comparison between uncorrected and CBSI-transformed data in the absence of short-distance channels to isolate the effects of blood flow in the scalp, see Pfeifer et al., ${ }^{21}$ for similar approach.

From a superficial perspective, our data analyses suggested that fNIRS data transformation by CBSI improved the sensitivity of the original data but that would be an oversimplification and a number of important caveats must be noted. The CBSI function was developed originally to correct for positive correlations between $\mathrm{HbO}$ and $\mathrm{HHb}$ caused by head movement $\mathrm{t}^{32}$ and designed to be used in real-time analyses. ${ }^{39}$ Cui et al. ${ }^{32}$ also reported that the CBSI transformation reduced spikes in the data, modulated nonspike noise to improve signal quality (see Ref. 40 for supporting evidence), and improved spatial specificity of the signals. According to one recent paper, ${ }^{41}$ there are five causes of positive association between $\mathrm{HbO}$ and $\mathrm{HHb}$ in the fNIRS signal, these are: (i) motion and head movement, (ii) cross-talk between the chromophores, (iii) differences in spatial responses due to the density of capillaries compared with large blood vessels, (iv) systemic effects from extracerebral sources, and (v) effects related to oxygenation and hemodynamic effects associated with muscle activation. In the case of our studies, there is an open question as to which of these factors may have been mitigated by the CBSI transformation. We would argue that (i) can be disregarded because participants were seated in a comfortable position, looking straight at the screen and significant levels of head movement were not observed. The influence of chromophore cross-talk (ii) is also unlikely because the fNIRS device utilized wavelength pairs that were optimized for measurement $(730$ and $850 \mathrm{~nm})$. The issue described by (iii) could account for individual variation but is unlikely to explain the prevalence of the positive association between $\mathrm{HbO}$ and $\mathrm{HHb}$ observed in study one. With respect to (iv), evidence for motivational intensity theory has been derived from changes in cardiovascular psychophysiology, specifically an increase of beta-adrenergic activity in the sympathetic branch of the autonomic nervous system. ${ }^{37,42}$ It is likely that changes in blood pressure and heart rate triggered by the cognitive demands of the task exerted an influence on cerebral blood flow and volume. It is also possible that autonomic changes exerted their influence on extracerebral sources such as blood flow in the scalp, ${ }^{20}$ for example, Takahashi et al., ${ }^{43}$ measured skin blood flow at the forehead and observed an increase in both pulse rate and amplitude during a verbal fluency test. ${ }^{44}$ The fifth possibility of hemodynamic activation related to muscle activation has been explored with respect to influence of teeth clenching on activation of temporal muscle $e^{41,44}$ and subsequent fNIRS measurement at anterior temporal sites. In the case of our studies, the fNIRS probe was positioned directly over the forehead and the frontalis muscle. This is significant because activation of the frontalis has been associated with mental effort in response to increased time pressure, ${ }^{45}$ the capacity to sustain cognitive performance over time, ${ }^{46}$ and increased mental workload. ${ }^{47}$ It is reasonable to expect that high levels of working memory load would increase activation of frontalis muscle with respect to blood flow and oxygenation. Of the five possible causes of positive association between $\mathrm{HbO}$ and $\mathrm{HHb}$ in our two experiments, a combination of increased sympathetic dominance and activation of the frontalis muscle would appear to be the most likely sources for the positive association between $\mathrm{HbO}$ and HHb shown in Figs. 2 and 3.

The CBSI transformation was effective with respect to the removal of positive correlation between $\mathrm{HbO}$ and $\mathrm{HHb}$. This approach compares favorably with other methods of artifact correction (e.g., PCA, wavelet filtering, spline interpolation, and Kalman filtering) ${ }^{48}$ However, Brigadoi et al., ${ }^{48}$ made the point that the CBSI transformation is reliant upon a number of assumptions, i.e., positive correlation between $\mathrm{HbO}$ and $\mathrm{HHb}$ is always defined as an artifact and the ratio of oxygenated to deoxygenated hemoglobin is assumed to be constant. Violation of either assumption would invalidate the approach, more importantly, they highlight the fact that $\mathrm{HHb}$ is largely derived from $\mathrm{HbO}$ within the CBSI formula, creating high dependence of one signal on the other. For example, if we consider the patterns of HbO_CBSI and HHb observed in study two and shown in Figs. 4 and 6, both measures seem to mirror one another. This observation begs a number of questions, to what extent is the pattern of $\mathrm{HHb}$ _CBSI simply the converse of $\mathrm{HbO}$ ? And if so, should those effects observed for HHb_CBSI be attributed to changes in the original $\mathrm{HbO}$ data? This entanglement is especially problematic for ascribing causal effects when more than one independent variable is included in the experimental design. This feature of the CBSI transformation requires further exploration with additional measures to assess the impact of intervening variables, e.g., short leads to assess scalp blood 
flow, cardiovascular impedance to assess sympathetic activation, and a sophisticated approach to statistical analyses, such as multilevel modeling. ${ }^{49}$

In conclusion, both studies provided support for the predictions of motivational intensity theory from neurovascular activation in the prefrontal cortex. In addition, there was evidence that the CBSI transformation removed a positive association between $\mathrm{HbO}$ and $\mathrm{HHb}$ and delivered a pattern of results consistent with a priori predictions of a psychological model. However, there is potential for the CBSI transformation to distort fNIRS data, with levels of HHb being particularly susceptible, and this aspect of the approach requires exploration through further research.

\section{Disclosures}

No conflicts of interest, financial or otherwise, are declared by the authors.

\section{Acknowledgments}

This work was funded by the BIAL Foundation as part of the project 46/12 "Motivational intensity in the prefrontal cortex."

\section{References}

1. M. Ferrari and V. Quaresima, "A brief review on the history of human functional near-infrared spectroscopy (fNIRS) development and fields of application," Neuroimage 63(2), 921-935 (2012).

2. G. E. Strangman, Z. Li, and Q. Zhang, "Depth sensitivity and sourcedetector separations for near infrared spectroscopy based on the Colin27 brain template," PLoS One 8(8), e66319 (2013)

3. N. Ramnani and A. M. Owen, "Anterior prefrontal cortex: insights into function from anatomy and neuroimaging," Nat. Rev. Neurosci. 5, 184-194 (2004).

4. H. Ayaz et al., "Optical brain monitoring for operator training and mental workload assessment," Neuroimage 59(1), 36-47 (2012).

5. C. Herff et al., "Mental workload during n-back task-quantified in the prefrontal cortex using fNIRS," Front. Hum. Neurosci. 7, 935 (2014).

6. F. A. Fishburn et al., "Sensitivity of fNIRS to cognitive state and load," Front. Hum. Neurosci. 8, 76 (2014).

7. U. León-Domínguez, J. F. Martín-Rodríguez, and J. León-Carrión, "Executive n-back tasks for the neuropsychological assessment of working memory," Behav. Brain Res. 292, 167-173 (2015).

8. E. Molteni et al., "Activation of the prefrontal cortex during a visual n-back working memory task with varying memory load: a near infrared spectroscopy study," Conf. Proc. IEEE Eng. Med. Biol. Soc. 2008, 4024-4027 (2008).

9. G. Durantin et al., "Using near infrared spectroscopy and heart rate variability to detect mental overload," Behav. Brain Res. 259, 16-23 (2014).

10. H. Ayaz et al., "Continuous monitoring of brain dynamics with functional near infrared spectroscopy as a tool for neuroergonomic research: empirical examples and a technological development," Front. Hum. Neurosci. 7, 871 (2013).

11. M. Niaz and R. H. Logie, "Working memory, mental capacity and science education: towards an understanding of the "working memory overload hypothesis'," Oxford Rev. Educ. 19(4), 511-525 (2006).

12. A. Kramer and J. Spinks, "Capacity views of human information processing," in Handbook of Cognitive Psychophysiology: Central and Nervous Systems Approaches, J. R. Jennings and M. G. H. Coles, Eds., pp. 179-249, Wiley, New York (1991).

13. J. W. Brehm and E. A. Self, "The intensity of motivation," Annu. Rev. Psychol. 40, 109-131 (1989).

14. R. A. Wright, "Refining the prediction of effort: Brehm's distinction between potential motivation and motivation intensity," Soc. Pers. Psychol. Compass 2(2), 682-701 (2008).

15. M. Richter, G. H. E. Gendolla, and R. A. Wright, "Three decades of research on motivational intensity theory: what we have learned about effort and what we still don't know," in Advances in Motivation Science, A. J. Elliot, Ed., pp. 149-186, Elsevier, San Diego, California (2016).
16. S. H. Fairclough and K. Ewing, "The effect of task demand and incentive on neurophysiological and cardiovascular markers of effort," Int. J. Psychophysiol. 119, 58-66 (2017).

17. F. Scholkmann et al., "A review on continuous wave functional nearinfrared spectroscopy and imaging instrumentation and methodology," Neuroimage 85 (Pt1), 6-27 (2014).

18. P. J. Gianaros, F. M. Van der Veen, and J. R. Jennings, "Regional cerebral blood flow correlates with heart period and high-frequency heart period variability during working-memory tasks: Implications for the cortical and subcortical regulation of cardiac autonomic activity," Psychophysiology 41(4), 521-530, (2004).

19. F. Scholkmann et al., "End-tidal $\mathrm{CO}_{2}$ : an important parameter for a correct interpretation in functional brain studies using speech tasks," Neuroimage 66, 71-79 (2013).

20. I. Tachtsidis and F. Scholkmann, "False positives and false negatives in functional near-infrared spectroscopy: issues, challenges, and the way forward," Neurophotonics 3(3), 031405 (2016).

21. M. D. Pfeifer, F. Scholkmann, and R. Labruyère, "Signal processing in functional near-infrared spectroscopy (fNIRS): methodological differences lead to different statistical results," Front. Hum. Neurosci. 11, 641 (2018).

22. G. Bauernfeind et al., "Separating heart and brain: on the reduction of physiological noise from multichannel functional near-infrared spectroscopy (fNIRS) signals," J. Neural Eng. 11, 056010 (2014).

23. M. Amian, S. K. Setarehdan, and H. Yousefi, "An ARMA model based motion artifact reduction algorithm in fNIRS data through a Kalman filtering approach," Proc. SPIE 9216, 921614 (2014).

24. D. Bontrager et al., "Physiological noise cancellation in fNIRS using an adaptive filter based on mutual information," in IEEE Int. Conf. on Systems, Man, and Cybernetics (SMC), San Diego, California, pp. 2014 (2014).

25. F. B. Haeussinger et al., "Reconstructing functional near-infrared spectroscopy (fNIRS) signals impaired by extra-cranial confounds: an easy-to-use filter method," Neuroimage 95, 69-79 (2014).

26. E. Kirilina et al., "The physiological origin of task-evoked systemic artefacts in functional near infrared spectroscopy," Neuroimage 61(1), 70-81 (2012).

27. M. A. Yucel et al., "Targeted principle component analysis: a new motion artifact correction approach for near-infrared spectroscopy," J. Innov. Opt. Health Sci. 7(2), 1350066 (2014).

28. E. Kirilina et al., "Identifying and quantifying main components of physiological noise in functional near infrared spectroscopy on the prefrontal cortex," Front. Hum. Neurosci. 7, 864 (2013).

29. M. A. Kamran and K. S. Hong, "Reduction of physiological effects in fNIRS waveforms for efficient brain-state decoding," Neurosci. Lett. 580, 130-136 (2014).

30. T. Yamada, S. Umeyama, and K. Matsuda, "Separation of fNIRS signals into functional and systemic components based on differences in hemodynamic modalities," PLoS One 7(11), e50271 (2012).

31. M. A. Kamran, M. M. Mannan, and M. Y. Jeong, "Cortical signal analysis and advances in functional near-infrared spectroscopy signal: a review," Front. Hum. Neurosci. 10, 261 (2016).

32. X. Cui, S. Bray, and A. L. Reiss, "Functional near infrared spectroscopy (NIRS) signal improvement based on negative correlation between oxygenated and deoxygenated hemoglobin dynamics," Neuroimage 49(4), 3039-3046 (2010).

33. R. B. Buxton, E. C. Wong, and L. R. Frank, "Dynamics of blood flow and oxygenation changes during brain activation: the Ballon model," Magn. Reson. Med. 39(6), 855-864 (1998).

34. Q. Luo et al., "Monitoring of brain activity with near-infrared spectroscopy," in Optical Biomedical Diagnostics., V. V. Tuchin, Ed., SPIE Press, Bellingham, Washington (2002).

35. H. Ayaz et al., "Sliding-window motion artifact rejection for functional near-infrared spectroscopy," in 32nd Annual Int. Conf. of the IEEE Engineering in Medicine and Biology Society, Buenos Aires, IEEE (2010).

36. L. Eubanks, R. A. Wright, and B. J. Williams, "Reward influence on the heart: cardiovascular response as a function of incentive value at five levels of task demand," Motiv. Emotion 26(2), 139-152 (2002).

37. M. Richter and G. H. E. Gendolla, "The heart contracts to reward: monetary incentives and preejection period," Psychophysiology 46, 451-457 (2009). 
38. A. M. Owen et al., "N-back working memory paradigm: a meta-analysis of normative functional neuroimaging studies," Hum. Brain Mapp. 25, 46-59 (2005).

39. M. Luhrs and R. Goebel, "Turbo-Satori: a neurofeedback and brain-computer interface toolbox for real-time functional near-infrared spectroscopy," Neurophotonics 4(4), 041504 (2017).

40. F. S. Racz et al., "Increased prefrontal cortex connectivity during cognitive challenge assessed by fNIRS imaging," Biomed. Opt. Express 8(8), 3842-3855 (2017).

41. G. A. Zimeo Morais et al., "Non-neuronal evoked and spontaneous hemodynamic changes in the anterior temporal region of the human head may lead to misinterpretations of functional near-infrared spectroscopy signals," Neurophotonics 5(1), 011002 (2018).

42. M. Richter, A. Friedrich, and G. H. Gendolla, "Task difficulty effects on cardiac activity," Psychophysiology 45, 869-875 (2008).

43. T. Takahashi, Y. Takikawa, and R. Kawagoe, "Differences in the pulsatile component of the skin hemodynamic response to verbal fluency tasks in the forehead and the fingertip," Sci. Rep. 6, 20978 (2016).

44. M. Schecklmann et al., "The temporal muscle of the head can cause artifacts in optical imaging studies with functional near-infrared spectroscopy," Front. Hum. Neurosci. 11, 456 (2017).

45. A. Van Boxtel and M. Jessurun, "Amplitude and bilateral coherency of facial and jaw-elevator EMG activity as an index of effort during a twochoice serial reaction task," Psychophysiology 30(6), 589-604 (1993).

46. M. Waersted and R. H. Westgaard, "Attention-related muscle activity in different body regions during VDU work with minimal physical activity," Ergonomics 39(4), 661-676 (1996).

47. J. Mead et al., "Investigating facial electromyography as an indicator of cognitive workload," in 19th Int. Symp. on Aviation Psychology (ISAP) (2017).
48. S. Brigadoi et al., "Motion artifacts in functional near-infrared spectroscopy: a comparison of motion correction techniques applied to real cognitive data," Neuroimage 85 (Pt1), 181-191 (2014).

49. F. Orihuela-Espina et al., "Quality control and assurance in functional near infrared spectroscopy (fNIRS) experimentation," Phys. Med. Biol. 55(13), 3701-3724 (2010).

Stephen H. Fairclough is a professor of psychophysiology at Liverpool John Moores University. His research is focused on development of applied methods for fNIRS analyses in the fields of neuroergonomics and human-computer interaction. His research on physiological computing has resulted in the publication of three edited collections and four special journal issues on this topic.

Christopher Burns is a postdoctoral research fellow at Warwick University's WMG engineering faculty in experiential engineering. Currently, he works on aspects of the UK Autodrive project, investigating human factors and psychophysiological responses arising from passenger experiences and pedestrian interactions with autonomously driven vehicles.

Ute Kreplin is a lecturer in biological psychology at Massey University in New Zealand. Her research is concerned with the understanding of prefrontal cortex activation during the experience of emotion. She has a keen interest in the use of functional near-infrared spectroscopy for neuropsychological research and implications of data analysis and application in this context. Her work has resulted in a number of publications. 